\title{
ROBUST NO ARBITRAGE AND THE SOLVABILITY OF VECTOR-VALUED UTILITY MAXIMIZATION PROBLEMS
}

\author{
ANDREAS H. HAMEL, BIRGIT RUDLOFF, AND ZHOU ZHOU
}

\begin{abstract}
A market model with $d$ assets in discrete time is considered where trades are subject to proportional transaction costs given via bid-ask spreads, while the existence of a numéraire is not assumed. It is shown that robust no arbitrage holds if, and only if, there exists a Pareto solution for some vector-valued utility maximization problem with component-wise utility functions. Moreover, it is demonstrated that a consistent price process can be constructed from the Pareto maximizer.
\end{abstract}

\section{INTRODUCTION}

The fundamental theorem of asset pricing (FTAP) has been studied extensively in various settings. In the FTAP, the equivalence of no arbitrage (NA) and the existence of an equivalent (super-)martingale measure is established. In market models with transaction costs, the martingale measures are usually replaced by consistent pricing systems (or processes). We refer to [1] and the references therein. There is also a stream of literature (e.g., [2, 7, 8, and the references therein) on general utility maximization with the presence of a numéraire in the market. In many papers, the existence of solutions (maximizers) is established, and one of the common assumptions is the absence of arbitrage. The easy-to-see fact that the converse is also true is well-known as well, i.e., the existence of a utility maximizer implies NA. For example, in [3, Section 3.1], the authors show both directions for a one-period market model without transaction costs which involves a numéraire. They show that no arbitrage is equivalent to the existence of a solution for some utility maximization problem. This result goes back to Rogers [10] who actually constructed equivalent martingale measures from utility maximizers.

The aim of this paper is to provide a corresponding result for market models with proportional transaction costs and a utility maximization problem with component-wise utility functions. This equivalence result between the existence of Pareto maximizers of a such vector-valued utility maximization problem and the well-established robust no-arbitrage condition (introduced in [13]) underlines that from a mathematical point of view the very special case of "one utility function for each asset" is already enough. Moreover, there is some rational to this situation: the interpretation is that the market model rules the exchange of assets, while "utilities" (the images of a vector-valued utility function) cannot be exchanged into one another and are therefore compared by means of the order generated by $\mathbb{R}_{+}^{d}$ and not a solvency cone (which is usually bigger). It also seems more plausible to have a complete preference for positions of only one asset-instead of a complete preference

Date: September 4, 2019.

Key words and phrases. Bid-ask spread, robust no arbitrage, Pareto maximizer, utility maximization, consistent price process. 
for portfolio vectors as assumed very often in the literature. Compare [4] where this approach was initiated.

A financial market in discrete time is considered in which there are $d$ tradable assets. The existence of a numéraire is not assumed; instead, following Kabanov [5], the assets are considered in physical units where the exchange between these assets are subject to proportional transaction costs. We show that robust no arbitrage holds if, and only if, there exists a solution for some vector-valued utility maximization problem with respect to the order generated by $\mathbb{R}_{+}^{d}$ (a Pareto maximizer). A key step to prove the result is to apply Komlós's theorem to get the closedness of the set of the vector-valued expected utilities. We also show that a consistent price process can be constructed from the Pareto maximizer.

The note is organized as follows. In the next section, we introduce the setup and the main results. In Section 3, we provide the proofs of the results.

\section{The SETUP AND THE MAIN RESUlT}

2.1. Notation and background. We follow the setup in [13. Let $\left(\Omega,\left(\mathcal{F}_{t}\right)_{t=0, \ldots, T}, \mathbb{P}\right)$ be a filtered probability space in discrete time, where $T \in \mathbb{N}$ is the time horizon, and $\mathcal{F}_{0}$ is assumed to be trivial. We assume that in the market there are $d$ assets available for exchange. These assets could be interpreted as currencies, but this restriction is not necessary. The exchange of assets is subject to proportional transaction costs; we use it in the form of bid-ask processes as discussed in [13].

Definition 2.1. A $d \times d$-matrix $\Pi_{0}=\left(\pi^{i j}\right)_{i, j=1, \ldots, d}$ is called a bid-ask matrix, if

$$
\pi^{i j}>0, \quad \pi^{i i}=1, \quad \text { and } \quad \pi^{i j} \leq \pi^{i k} \pi^{k j}, \quad \forall i, j, k=1, \ldots, d .
$$

An adapted process $\Pi=\left(\Pi_{t}\right)_{t=0, \ldots, T}$ is called a bid-ask process, if $\Pi_{t}(\omega)$ is a bid-ask matrix for any $(t, \omega) \in\{0, \ldots, T\} \times \Omega$.

In the above definition, the entry $\pi_{t}^{i j}$ in $\Pi_{t}$ denotes the number of units of asset $i$ an investor needs in order to buy one unit of asset $j$ at time $t$. That is, the bid-ask spread of asset $i$ in terms of asset $j$ is given by $\left\{1 / \pi_{t}^{j i}, \pi_{t}^{i j}\right\}$ at time $t$.

For the bid-ask matrix $\Pi_{0}=\left(\pi_{i j}\right)$, let $-K\left(\Pi_{0}\right)$ be the convex cone generated by the vectors $\left\{-e^{i},-\pi^{i j} e^{i}+e^{j}, i, j=1, \ldots, d\right\}$, where $e^{i}=(0, \ldots, 0,1,0, \ldots, 0)$ with the $i$-th component equal to 1 . In other words, $-K\left(\Pi_{0}\right)$ represents the set of portfolios available starting from initial position $0 \in \mathbb{R}^{d}$ for the assets. Denote $K^{*}\left(\Pi_{0}\right)$ as the polar of the cone $-K\left(\Pi_{0}\right)$. That is,

$$
K^{*}\left(\Pi_{0}\right):=\left\{w \in \mathbb{R}^{d}:\langle v, w\rangle \leq 0, \forall v \in-K\left(\Pi_{0}\right)\right\},
$$

where $\langle\cdot, \cdot\rangle$ represents the inner product. For any bid-ask process $\Pi=\left(\Pi_{t}\right)_{t=0, \ldots, T}$, similar definitions apply for $-K\left(\Pi_{t}(\omega)\right)$ and $K^{*}\left(\Pi_{t}(\omega)\right),(t, \omega) \in\{0, \ldots, T\} \times \Omega$.

Let us recall the definition of self-financing portfolios in [13].

Definition 2.2. An $\mathbb{R}^{d}$-valued adapted process $v=\left(v_{t}\right)_{t=0, \ldots, T}$ is called a self-financing portfolio process (from initial position 0 ) for the bid-ask process $\Pi=\left(\Pi_{t}\right)_{t=0, \ldots, T}$, if for $t=0, \ldots, T$,

$$
v_{t}-v_{t-1} \in-K\left(\Pi_{t}\right), \quad \text { a.s. }
$$


where $v_{-1} \equiv 0$. Denote

$$
A_{\Pi}:=\left\{v_{T}: \text { for some self-financing portfolio process }\left(v_{t}\right)_{t=0, \ldots, T} \text { in terms of } \Pi\right\} .
$$

Recall the notion of (robust) no arbitrage introduced in [13].

Definition 2.3. We say $N A$ holds with respect to the bid-ask process $\Pi$, if for any $v_{T} \in A_{\Pi}$, if $v_{T} \geq 0$ a.s. then $v_{T}=0$ a.s., where the (in)equalities are component-wise. We say $N A^{r}$ holds with respect to $\Pi$, if there exists a bid-ask process $\tilde{\Pi}=\left(\tilde{\Pi}_{t}\right)_{t}$ with smaller bid-ask spreads (i.e., for $t=0, \ldots, T$ and $i, j=1, \ldots, d,\left[1 / \tilde{\pi}_{t}^{j i}, \tilde{\pi}_{t}^{i j}\right]$ is contained in the relative interior of $\left[1 / \pi_{t}^{j i}, \pi_{t}^{i j}\right]$ a.s.), such that $N A$ holds with respect to $\tilde{\Pi}$.

Let us also recall the definition of (strictly) consistent price processes.

Definition 2.4. An $\mathbb{R}_{+}^{d}$ valued adapted process $Z=\left(Z_{t}\right)_{t=0, \ldots, T}$ is called a consistent (strictly consistent, respectively) price process for the bid-ask process $\Pi$, if $Z$ is a $\mathbb{P}$-martingale, and $Z_{t}(\cdot) \in$ $K_{t}^{*}(\cdot) \backslash\{0\}$ (the relative interior of $K_{t}^{*}(\cdot)$, respectively) a.s., $t=0, \ldots, T$.

For $i=1, \ldots, d$, let $U^{i}: \mathbb{R}_{+} \mapsto[-\infty, \infty)$ be concave, continuous, strictly increasing, and bounded from above, representing the utility function for asset $i$. Define $U: \mathbb{R}_{+}^{d} \mapsto \mathbb{R}^{d}$,

$$
U(x)=\left(U^{1}\left(x^{1}\right), \ldots, U^{d}\left(x^{d}\right)\right), \quad x=\left(x^{1}, \ldots, x^{d}\right) \in \mathbb{R}_{+}^{d} .
$$

For a given initial endowment $x \in \mathbb{R}_{+}^{d}$, consider the $\mathbb{R}^{d}$-valued utility maximization problem

$$
\text { maximize } \mathbb{E}_{\mathbb{P}} U\left(X_{T}\right) \text { subject to } X_{T} \in \mathcal{A}_{\Pi}(x),
$$

where

$$
\mathcal{A}_{\Pi}(x):=\left\{X_{T} \geq 0 \text { a.s. : } X_{T} \in A_{\Pi}+x\right\}
$$

and

$$
\mathbb{E}_{\mathbb{P}} U\left(X_{T}\right):=\left(\mathbb{E}_{\mathbb{P}} U^{1}\left(X_{T}^{1}\right), \ldots, \mathbb{E}_{\mathbb{P}} U^{d}\left(X_{T}^{d}\right)\right), \quad X_{T}=\left(X_{T}^{1}, \ldots, X_{T}^{d}\right) \in \mathcal{A}_{\Pi}(x) .
$$

Moreover, we also consider the set

$$
J_{\Pi}(U)(x):=\left\{\mathbb{E}_{\mathbb{P}} U\left(X_{T}\right): X_{T} \in A_{\Pi}(x)\right\} .
$$

The meaning of the word "maximize" in (2.1) has to be defined. We will consider two variants. First, we recall the definition of a Pareto maximizer with respect to the ordering cone $\mathbb{R}_{+}^{d}$ and a domination property which is also called (upper) external stability in the literature. For yet another notion, compare Remark 2.9 below.

Definition 2.5. (a) A point $\hat{z} \in M$ is called Pareto maximal for $M \subseteq \mathbb{R}^{d}$ iff

$$
M \cap\left(\hat{z}+\mathbb{R}_{+}^{d}\right)=\{\hat{z}\} .
$$

The set of Pareto maximal points of $M$ is denoted by $\operatorname{Max} M$.

(b) A set $M \subseteq \mathbb{R}^{d}$ is said to satisfy the upper domination property if for each $z \in M$ there is $\hat{z} \in \operatorname{Max} M$ such that $z \leq_{\mathbb{R}_{+}^{d}} \hat{z}$. 
Definition 2.6. A position $\hat{X}_{T} \in \mathcal{A}_{\Pi}(x)$ is called a Pareto maximizer for (2.1) iff $\mathbb{E}_{\mathbb{P}} U\left(\hat{X}_{T}\right) \in$ $\operatorname{Max} J_{\Pi}(U)(x)$. The set of Pareto maximizers for (2.1) is denoted by $P_{\Pi}(U)(x)$.

One may easily realize that $\hat{X}_{T} \in P_{\Pi}(U)(x)$ if, and only if, there does not exist $X_{T} \in \mathcal{A}_{\Pi}(x)$, such that

i) For all $i \in\{1, \ldots, d\}, \mathbb{E}_{\mathbb{P}} U^{i}\left(X_{T}^{i}\right) \geq \mathbb{E}_{\mathbb{P}} U^{i}\left(\hat{X}_{T}^{i}\right)$;

ii) For some $i \in\{1, \ldots, d\}, \mathbb{E}_{\mathbb{P}} U^{i}\left(X_{T}^{i}\right)>\mathbb{E}_{\mathbb{P}} U^{i}\left(\hat{X}_{T}^{i}\right)$.

The study of the vector-valued utility maximization problem (2.1) was initiated in [14] and [15], the former containing a certainty equivalent, the latter duality results in a one-period setup with a finite probability space which were published in [4]. In [16], the problem is studied with each of the components of the utility function depending on all the components of the commodity vector in a deterministic and set-valued framework. In [12], further certainty equivalents as well as indifference prices are defined also for a finite probability space.

2.2. Main results. The following two results, Proposition 2.7 and Theorem 2.8, establish the equivalence between no arbitrage and the existence of a Pareto optimizer for (2.1).

Proposition 2.7. Let $\Pi$ be a bid-ask process. If $N A^{r}$ holds with respect to $\Pi$, then $J_{\Pi}(U)(x)$ satisfies the upper domination property. In particular, $P_{\Pi}(U) \neq \emptyset$.

Conversely, if $P_{\Pi}(U) \neq \emptyset$, then $N A$ holds with respect to $\Pi$.

Theorem 2.8. $N A^{r}$ holds with respect to the bid-ask process $\Pi$ if, and only if, there exists a bid-ask process $\tilde{\Pi}$ with smaller bid-ask spreads satisfying $P_{\tilde{\Pi}}(U) \neq \emptyset$.

Remark 2.9. A comment on the above results might be in order. The function $X_{T} \mapsto \mathbb{E}_{\mathbb{P}} U\left(X_{T}\right)$ can straightforwardly be extended to a function $F$ mapping into the set $\mathcal{G}\left(\mathbb{R}^{d},-\mathbb{R}_{+}^{d}\right):=\left\{D \subseteq \mathbb{R}^{d}: D=\right.$ $\left.\operatorname{clco}\left(D-\mathbb{R}_{+}^{d}\right)\right\}$ by setting

$$
F\left(X_{T}\right)=\mathbb{E}_{\mathbb{P}} U\left(X_{T}\right)-\mathbb{R}_{+}^{d} .
$$

The pair $\left(\mathcal{G}\left(\mathbb{R}^{d},-\mathbb{R}_{+}^{d}\right), \subseteq\right)$ is a complete lattice. The first part of Proposition 2.7 can now be read as follows: $N A^{r}$ implies the existence of a solution of the set optimization problem (in the completelattice sense, see, e.g., [11, Definition 28, Corollary 2.9])

$$
\text { maximize } F\left(X_{T}\right) \text { subject to } X_{T} \in \mathcal{A}_{\Pi}(x) \text {. }
$$

This follows from [11, Proposition 2.15] (adapted to maximization), and in this reference more material about the treatment of vector optimization as complete lattice-valued problems can be found.

Thus, roughly speaking, Theorem 2.8 yields that robust no arbitrage is equivalent to the existence of solutions of the complete lattice extension of some vector-valued utility maximization problem. One should note that the existence of such a solution does not imply the domination property.

Motivated by [3, Corollary 3.10], we also establish the following wo results which provide a construction of a (strictly) consistent price process from a Pareto maximizer. 
Proposition 2.10. Let $N A^{r}$ hold with respect to the bid-ask process $\Pi$ and let $\hat{X}_{T} \in P_{\Pi}(U)$. Assume that $U^{i}$ is differentiable for all $i=1, \ldots, d$, and that there exists $\delta \in \operatorname{int} \mathbb{R}_{+}^{d}$ such that $\hat{X}_{T} \geq \delta$ a.s. Then, there exists $\left(\lambda^{1}, \ldots, \lambda^{d}\right) \in \mathbb{R}_{+}^{d} \backslash\{0\}$ such that

$$
\left(\left(\lambda^{i} \mathbb{E}_{\mathbb{P}}\left[\left(U^{i}\right)^{\prime}\left(\hat{X}_{T}^{i}\right) \mid \mathcal{F}_{t}\right]\right)_{i=1, \ldots, d}\right)_{t=0, \ldots, T}
$$

defines a consistent price process for $\Pi$.

Corollary 2.11. Let $N A^{r}$ hold with respect to the bid-ask process $\Pi$, and thus $N A^{r}$ still holds with respect to some $\tilde{\Pi}$ with smaller bid-ask spreads. Let $\hat{X}_{T} \in P_{\tilde{\Pi}}(U)$. Assume that $U^{i}$ is differentiable for all $i=1, \ldots, d$, and that there exists $\delta \in \operatorname{int} \mathbb{R}_{+}^{d}$ such that $\hat{X}_{T} \geq \delta$ a.s. Then, there exists $\left(\lambda^{1}, \ldots, \lambda^{d}\right) \in \operatorname{int} \mathbb{R}_{+}^{d}$ such that

$$
\left(\left(\lambda^{i} \mathbb{E}_{\mathbb{P}}\left[\left(U^{i}\right)^{\prime}\left(\hat{X}_{T}^{i}\right) \mid \mathcal{F}_{t}\right]\right)_{i=1, \ldots, d}\right)_{t=0, \ldots, T}
$$

defines a strictly consistent price process for $\Pi$.

\section{Proof of the Results}

Lemma 3.1. Assume $N A^{r}$ holds with respect to $\Pi$. Then for any sequence $\left(X_{T}^{n}\right)_{n \in \mathbb{N}} \subset \mathcal{A}_{\Pi}(x)$, there exists a subsequence $\left(X_{T}^{n_{k}}\right)_{k \in \mathbb{N}} \subset\left(X_{T}^{n}\right)_{n \in \mathbb{N}}$ and $Y \in \mathcal{A}_{\Pi}(x)$, such that

$$
\frac{1}{N} \sum_{k=1}^{N} X_{T}^{n_{k}} \rightarrow Y \text { a.s. }, \quad N \rightarrow \infty .
$$

Proof. By [13, Theorem 1.7], there exists a strictly consistent price process $\left(Z_{t}\right)_{t=0, \ldots, T}$. For $i=$ $1, \ldots, d$, define

$$
\frac{d \mathbb{Q}^{i}}{d \mathbb{P}}:=\frac{Z_{T}^{i}}{Z_{0}^{i}}
$$

Then, $\mathbb{Q}^{i}$ is a probability measure that is equivalent to $\mathbb{P}, i=1, \ldots, d$. For any $X_{T}=\left(X_{T}^{1}, \ldots, X_{T}^{d}\right) \in$ $\mathcal{A}_{\Pi}(x)$, we have that for $i=1, \ldots, d$,

$$
\mathbb{E}_{\mathbb{Q}^{i}} X_{T}^{i}=\frac{1}{Z_{0}^{i}} \mathbb{E}_{\mathbb{P}}\left[Z_{T}^{i} X_{T}^{i}\right] \leq \frac{1}{Z_{0}^{i}} \mathbb{E}_{\mathbb{P}}\left\langle Z_{T}, X_{T}\right\rangle \leq \frac{1}{Z_{0}^{i}}\left\langle Z_{0}, x\right\rangle,
$$

where the second (in)equality follows from the fact that the components of $Z_{T}$ and $X_{T}$ are all nonnegative a.s., and the third (in)equality follows from [13, Theorem 4.1].

Now, let $\left(X_{T}^{n}\right)_{n \in \mathbb{N}} \subset \mathcal{A}_{\Pi}(x)$, where $X_{T}^{n}=\left(X_{T}^{n, 1}, \ldots, X_{T}^{n, d}\right)$. First, consider the first component, i.e., the sequence $\left(X_{T}^{n, 1}\right)_{n \in \mathbb{N}}$. Since from (3.1)

$$
\sup _{n \in \mathbb{N}} \mathbb{E}_{\mathbb{Q}} X_{T}^{n, 1} \leq \frac{1}{Z_{0}^{1}}\left\langle Z_{0}, x\right\rangle<\infty
$$

by Komlós's theorem (see e.g., [6, Theorem 1a]), there exists a subsequence $\left(X_{T}^{n_{k}, 1}\right)_{k \in \mathbb{N}} \subset\left(X_{T}^{n, 1}\right)_{n \in \mathbb{N}}$ and a random variable $Y^{1} \in \mathcal{F}_{T}$, such that for any further subsequence $\left(\eta^{j}\right)_{j \in \mathbb{N}} \subset\left(X_{T}^{n_{k}, 1}\right)_{k \in \mathbb{N}}$,

$$
\frac{1}{N} \sum_{j=1}^{N} \eta^{j} \rightarrow Y^{1} \text { a.s., } \quad N \rightarrow \infty .
$$


Next, consider the sequence $\left(X_{T}^{n_{k}, 2}\right)_{k \in \mathbb{N}} \subset\left(X_{T}^{n, 2}\right)_{n \in \mathbb{N}}$ for the second component. Applying Komlós's theorem again, we have that there exist a subsequence $\left(X_{T}^{n_{k_{l}}, 2}\right)_{l \in \mathbb{N}} \subset\left(X_{T}^{n_{k}, 2}\right)_{k \in \mathbb{N}}$ and $Y^{2} \in \mathcal{F}_{T}$, such that for any further subsequence $\left(\xi_{j}\right)_{j \in \mathbb{N}} \subset\left(X_{T}^{n_{k_{l}}, 2}\right)_{l \in \mathbb{N}}$,

$$
\frac{1}{N} \sum_{j=1}^{N} \xi^{j} \rightarrow Y^{2} \text { a.s., } \quad N \rightarrow \infty .
$$

Obviously, (3.2) still holds if we replace the $\left(X_{T}^{n_{k}, 1}\right)_{k \in \mathbb{N}}$ with $\left(X_{T}^{n_{k_{l}}, 1}\right)_{l \in \mathbb{N}}$. Repeating this process for the remaining components, we can finally get a subsequence $\left(\zeta_{i}\right)_{i \in \mathbb{N}} \subset\left(X_{T}^{n}\right)_{n \in \mathbb{N}}$ and an $\mathbb{R}^{d}$-valued random variable $Y=\left(Y^{1}, \ldots, Y^{d}\right) \in \mathcal{F}_{T}$, such that

$$
\frac{1}{N} \sum_{i=1}^{N} \zeta_{i} \rightarrow Y \text { a.s. }, \quad N \rightarrow \infty .
$$

Since $\mathcal{A}_{\Pi}(x)$ is convex, $\frac{1}{N} \sum_{i=1}^{N} \zeta_{i} \in \mathcal{A}_{\Pi}(x)$. By [13, Theorem 2.1], $\mathcal{A}_{\Pi}(x)$ is closed in $L^{0}\left(\Omega, \mathcal{F}_{T}, \mathbb{P} ; \mathbb{R}^{d}\right)$, and thus $Y \in \mathcal{A}_{\Pi}(x)$.

Proof of Proposition 2.7. First, let us show the first statement of this proposition. Assume that $\mathrm{NA}^{r}$ holds with respect to $\Pi$. It suffices to show that the set $J_{\Pi}(U)(x) \subset \mathbb{R}^{d}$ is closed. Indeed, if this is the case, then the set $\left(\mathbb{E}_{\mathbb{P}} U\left(X_{T}\right)+\mathbb{R}_{+}^{d}\right) \cap J_{\Pi}(U)(x)$ is compact for each $X_{T} \in \mathcal{A}_{\Pi}(x)$ since $U$ is component-wise bounded from above. Therefore, by [9, Theorem 2(i), page 489], there is a Pareto maximizer $\bar{X}_{T} \in \mathcal{A}_{\Pi}(x)$ satisfying $\mathbb{E}_{\mathbb{P}} U\left(X_{T}\right) \leq_{\mathbb{R}_{+}^{d}} \mathbb{E}_{\mathbb{P}} U\left(\bar{X}_{T}\right)$. In particular, $P_{\Pi}(U) \neq \emptyset$.

In order to show the closedness of $J_{\Pi}(U)(x)$, take $\left(X_{T}^{n}\right)_{n \in \mathbb{N}} \subset \mathcal{A}_{\Pi}(x)$, such that

$$
\mathbb{E}_{\mathbb{P}} U\left(X_{T}^{n}\right) \rightarrow a \in\left(\prod_{i=1}^{d}\left[U^{i}(0), \infty\right)\right) \cap \mathbb{R}^{d} .
$$

By Lemma 3.1, there exists a subsequence $\left(X_{T}^{n_{k}}\right)_{k \in \mathbb{N}}$ and $Y \in \mathcal{A}_{\Pi}(x)$, such that

$$
\frac{1}{N} \sum_{k=1}^{N} X_{T}^{n_{k}} \rightarrow Y \text { a.s., } \quad N \rightarrow \infty .
$$

As $U_{i}$ is concave, $i=1, \ldots, d$, we have that

$$
\mathbb{E}_{\mathbb{P}} U\left(\frac{1}{N} \sum_{k=1}^{N} X_{T}^{n_{k}}\right) \geq \frac{1}{N} \sum_{k=1}^{N} \mathbb{E}_{\mathbb{P}} U\left(X_{T}^{n_{k}}\right),
$$

where the inequality is component-wise. As $U$ is continuous and bounded from above, by Fatou's Lemma, one has

$$
\mathbb{E}_{\mathbb{P}} U(Y) \geq a .
$$

Because $\mathcal{A}_{\Pi}(x)=\left(\mathcal{A}_{\Pi}(x)-L^{0}\left(\Omega, \mathcal{F}_{T}, \mathbb{P} ; \mathbb{R}_{+}^{d}\right) \cap L^{0}\left(\Omega, \mathcal{F}_{T}, \mathbb{P} ; \mathbb{R}_{+}^{d}\right)\right.$ and $U^{i}$ is continuous for $i=1, \ldots, d$, there exists $\tilde{Y} \in \mathcal{A}_{\Pi}(x)$, such that $\mathbb{E}_{\mathbb{P}} U(\tilde{Y})=a$. This completes the proof of the first statement.

Next, let us prove the second statement. Let $\hat{X}_{T} \in \mathcal{A}_{\Pi}(x)$ be a Pareto maximizer for (2.1). If NA fails with respect to $\Pi$, then there would exist some $\bar{X}_{T}=\left(\bar{X}_{T}^{1}, \ldots, \bar{X}_{T}^{d}\right) \in A_{\Pi}$, such that

$$
\text { for any } i \in\{1, \ldots, d\}, \quad \bar{X}_{T}^{i} \geq 0 \text { a.s., }
$$


and

$$
\text { for some } i \in\{1, \ldots, d\}, \quad \mathbb{P}\left\{\bar{X}_{T}^{i}>0\right\}>0 \text {. }
$$

Then $\hat{X}_{T}+\bar{X}_{T} \in \mathcal{A}_{\Pi}(x)$, and

$$
\text { for any } i \in\{1, \ldots, d\}, \quad \mathbb{E}_{\mathbb{P}} U^{i}\left(\hat{X}_{T}^{i}+\bar{X}_{T}^{i}\right) \geq \mathbb{E}_{\mathbb{P}} U^{i}\left(\hat{X}_{T}^{i}\right),
$$

and

$$
\text { for some } i \in\{1, \ldots, d\}, \quad \mathbb{E}_{\mathbb{P}} U^{i}\left(\hat{X}_{T}^{i}+\bar{X}_{T}^{i}\right)>\mathbb{E}_{\mathbb{P}} U^{i}\left(\hat{X}_{T}^{i}\right) .
$$

This contradicts the Pareto optimality of $\hat{X}_{T}$.

Proof of Theorem 2.8. If $\mathrm{NA}^{r}$ holds with respect to $\Pi$, then there exists $\tilde{\Pi}$ with smaller bid-ask spreads, such that $\mathrm{NA}^{r}$ also holds with respect to $\tilde{\Pi}$. By Proposition 2.7 one has $P_{\tilde{\Pi}}(U) \neq \emptyset$.

Conversely, if $P_{\tilde{\Pi}}(U) \neq \emptyset$ for some $\tilde{\Pi}$ with smaller bid-ask spreads, then by Proposition 2.7 NA holds with respect to $\tilde{\Pi}$, and thus $\mathrm{NA}^{r}$ holds with respect to $\Pi$.

Proof of Proposition 2.10. Since $\hat{X}_{T}$ is a Pareto optimizer for (2.1), by [9, Proposition 9, page 497] there exists $\lambda=\left(\lambda^{1}, \ldots, \lambda^{d}\right) \in \mathbb{R}_{+}^{d} \backslash\{0\}$, such that $\hat{X}_{T}$ is also optimal for the scalar-valued maximization problem

$$
\sup _{X_{T} \in \mathcal{A}_{\Pi}(x)}\left\langle\lambda, \mathbb{E}_{\mathbb{P}} U\left(X_{T}\right)\right\rangle .
$$

Fix $t \in\{0, \ldots, T\}$. Let $u_{t}$ be an $\mathbb{R}^{d}$-valued $\mathcal{F}_{t}$-measurable random variable satisfying $u_{t}(\cdot) \in$ $-K\left(\Pi_{t}(\cdot)\right)$ and $u_{t}(\cdot) \geq-\delta / 2$ a.s. Since $\hat{X}_{T}+\epsilon u_{t} \in \mathcal{A}_{\Pi}(x)$ for any $\epsilon \in(0,1)$ and $\hat{X}_{T}$ is optimal for (3.3), we have that

$$
\left\langle\lambda, \mathbb{E}_{\mathbb{P}} U\left(\hat{X}_{T}+\epsilon u_{t}\right)\right\rangle-\left\langle\lambda, \mathbb{E}_{\mathbb{P}} U\left(\hat{X}_{T}\right)\right\rangle \leq 0
$$

Then

$$
\sum_{i=1}^{d} \lambda_{i} \mathbb{E}_{\mathbb{P}}\left[\frac{U^{i}\left(\hat{X}_{T}^{i}+\epsilon u_{t}^{i}\right)-U^{i}\left(\hat{X}_{T}^{i}\right)}{\epsilon}\right] \leq 0 .
$$

Letting $\epsilon \searrow 0$ and applying the dominated convergence theorem, we get that

$$
\sum_{i=1}^{d} \lambda_{i} \mathbb{E}_{\mathbb{P}}\left[u_{t}^{i} \cdot\left(U^{i}\right)^{\prime}\left(\hat{X}_{T}^{i}\right)\right] \leq 0 .
$$

Therefore,

$$
\mathbb{E}_{\mathbb{P}}\left[\sum_{i=1}^{d} \lambda_{i} \mathbb{E}_{\mathbb{P}}\left[\left(U^{i}\right)^{\prime}\left(\hat{X}_{T}^{i}\right) \mid \mathcal{F}_{t}\right] u_{t}^{i}\right] \leq 0 .
$$

Let us show that

$$
\left(\lambda_{1} \mathbb{E}_{\mathbb{P}}\left[\left(U^{1}\right)^{\prime}\left(\hat{X}_{T}^{1}\right) \mid \mathcal{F}_{t}\right], \ldots, \lambda_{d} \mathbb{E}_{\mathbb{P}}\left[\left(U^{d}\right)^{\prime}\left(\hat{X}_{T}^{d}\right) \mid \mathcal{F}_{t}\right]\right)(\cdot) \in K^{*}\left(\Pi_{t}(\cdot)\right), \quad \text { a.s. }
$$

Indeed, if the above fails to be true, then there exists an $\mathcal{F}_{t}$-measurable $\bar{w}_{t}(\cdot) \in-K\left(\Pi_{t}(\cdot)\right)$ such that

$$
\mathbb{P}\left\{\sum_{i=1}^{d}\left(\lambda_{i} \mathbb{E}_{\mathbb{P}}\left[\left(U^{i}\right)^{\prime}\left(\hat{X}_{T}^{i}\right) \mid \mathcal{F}_{t}\right] \cdot \bar{w}_{t}^{i}\right)>0\right\}>0
$$


Then there exists $c>0$ such that

$$
\mathbb{P}\left\{c \bar{w}_{t}>-\delta / 2, \sum_{i=1}^{d}\left(\lambda_{i} \mathbb{E}_{\mathbb{P}}\left[\left(U^{i}\right)^{\prime}\left(\hat{X}_{T}^{i}\right) \mid \mathcal{F}_{t}\right] \cdot c \bar{w}_{t}^{i}\right)>0\right\}>0 .
$$

Denote the set in the above as $B$. If we replace $u_{t}$ in (3.4) by $\tilde{w}_{t}:=c \bar{w}_{t} 1_{B}$, then the inequality (3.4) fails, which yields a contradiction.

Proof of Corollary 2.11. The result follows from Proposition 2.10 and [13, Proposition A.5].

Contribution of the authors. The first author conjectured the results of this paper (in form of Proposition 2.7 and Remark 2.9); this conjecture is already mentioned in [14, 15] supervised by the first author regarding the content. The equivalence problem for solutions of vector-utility functions and (robust) no arbitrage for markets with transaction costs was among the open problems given by the first author to the second for discussion at the AMS Mathematics Research Community 2015 in Financial Mathematics. The collaboration of the second and the third author at this summer school led to the proof of this conjecture.

Acknowledgement. The last two authors gratefully acknowledge the financial support by the National Science Foundation, Division of Mathematical Sciences, under Grant No. 1321794.

\section{REFERENCES}

[1] R. Cont, Encyclopedia of Quantitative Finance (4 Volumes), John Wiley \& Sons, 2010

[2] J. Cvitanić, W. Schachermayer, H. Wang, Utility maximization in incomplete markets with random endowment, Finance and Stochastics 5(2), 259-272, 2001

[3] H. Föllmer, A. Schied, Stochastic Finance. An Introduction in Discrete Time (extended edition) de Gruyter Studies in Mathematics Vol. 27, Walter de Gruyter \& Co. 2004

[4] A. H. Hamel, Q. (Sophie) Wang, A set optimization approach to utility maximization under transaction costs, Decisions in Economics and Finance 40(1-2), 257-275, 2017

[5] Y. M. Kabanov, Hedging and liquidation under transaction costs in currency markets, Finance and Stochastics 3(2), 237-248, 1999

[6] J. Komlós, A generalization of a problem of Steinhaus, Acta Math. Acad. Sci. Hungar. 18, 217-229, 1967

[7] D. Kramkov, W. Schachermayer, The asymptotic elasticity of utility functions and optimal investment in incomplete markets, Ann. Appl. Probab. 9(3), 904-950, 1999

[8] D. Kramkov, W. Schachermayer, Necessary and sufficient conditions in the problem of optimal investment in incomplete markets, Ann. Appl. Probab. 13(4), 1504-1516, 2003

[9] D. T. Luc, Pareto optimality, in: A. Chinchuluun, P. M. Pardalos, A. Migdalas, L. Pitsoulis (eds.), Pareto Optimality, Game Theory and Equilibria, Springer Optimization and Its Applications Vol. 17, pp. 481-515, Springer, 2008

[10] L.C.G. Rogers, Equivalent martingale measures and no-arbitrage, Stochastics 51(1-2), 41-49, 1994

[11] A. Löhne, Vector Optimization with Infimum and Supremum, Springer Science \& Business Media, 2011

[12] B. Rudloff, F. Ulus, Certainty equivalent and utility indifference pricing for incomplete preferences via convex vector optimization, arXiv preprint, arXiv:1904.09456, 2019

[13] W. Schachermayer, The fundamental theorem of asset pricing under proportional transaction costs in finite discrete time, Math. Finance 14(1), 19-48, 2004

[14] Q. (Sophie) Wang, Utility, Junior Project Thesis, Princeton University, 2010

[15] Q. (Sophie) Wang, The utility maximization problem in markets with transaction costs: a set-valued approach, Senior Thesis, Princeton University, 2011 
[16] Zamboni, Martina, Set-valued optimization and application to economics, Master Thesis, Universitá degli Studi di Verona, 2015

Faculty of Economics and Management, Free University of Bozen-Bolzano

E-mail address: Andreas.Hamel@unibz.it

Institute for Statistics and Mathematics, Vienna University of Economics and Business

E-mail address: birgit.rudloff@wu.ac.at

School of Mathematics and Statistics, University of Sydney

E-mail address: zhou.zhou@sydney.edu.au 